\title{
Letter by Dyverfeldt and Ebbers regarding article "Estimation of turbulent kinetic energy using 4D phase-contrast MRI: Effect of scan parameters and target vessel size"
}

\author{
Petter Dyverfeldt and Tino Ebbers
}

\author{
Journal Article
}

\section{Tweet}

N.B.: When citing this work, cite the original article.

Original Publication:

Petter Dyverfeldt and Tino Ebbers, Letter by Dyverfeldt and Ebbers regarding article "Estimation of turbulent kinetic energy using 4D phase-contrast MRI: Effect of scan parameters and target vessel size", Magnetic Resonance Imaging, 2016. 34(8), pp.1226-1226. http://dx.doi.org/10.1016/j.mri.2016.05.010

Copyright: Elsevier

http://www.elsevier.com/

Postprint available at: Linköping University Electronic Press

http://urn.kb.se/resolve?urn=urn:nbn:se:liu:diva-131572

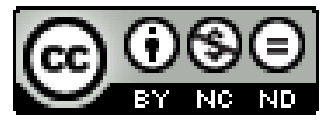



Manuscript type: Letter to the Editor

Title: Letter by Dyverfeldt and Ebbers Regarding Article "Estimation of Turbulent Kinetic Energy using 4D Phase-Contrast MRI: Effect of Scan Parameters and Target Vessel Size”

Authors: Petter Dyverfeldt ${ }^{\mathrm{a}, \mathrm{b}}$, Tino Ebbers ${ }^{\mathrm{a}, \mathrm{b}}$

${ }^{a}$ Division of Cardiovascular Medicine, Department of Medical and Health Sciences, Linköping University, Linköping, Sweden.

${ }^{\mathrm{b}}$ Center for Medical Image Science and Visualization, Linköping University, Linköping, Sweden.

Corresponding author address: PD. Linköping University Hospital, IMH/KVM/Klinfys, SE-581 83 Linköping, Sweden. E-mail: petter.dyverfeldt@liu.se

Keywords: MR Flow Imaging, 4D Flow MRI, Turbulence, Turbulent Kinetic Energy, VENC, Phase-Contrast 


\section{To the Editor:}

We read with great interest the recent article by Dr. Ha and colleagues about the effect of scan parameters and vessel size on turbulent kinetic energy (TKE) estimates obtained with 4D Flow MRI [1]. The study of Ha et al provides several valuable insights, including the findings that echo time and voxel size do not significantly influence measurements of the total TKE in a volume of interest. We are pleased that the interest in TKE mapping increases and that more laboratories are investigating the potential and limits of this intriguing MR flow imaging method.

Ha et al conclude that the velocity encoding range (VENC) setting can introduce bias when quantifying the total TKE. This conclusion appears to be based on the result that the $\operatorname{TKE}_{\text {sum }}[\mathrm{J}]$, as obtained by integrating the TKE per voxel $\left[\mathrm{J} / \mathrm{m}^{3}\right]$ in a region of interest, increases with increasing VENC (Figure 3b in the study by $\mathrm{Ha}$ et al). This contradicts with theory, which predicts there should be no such bias [2].

The total TKE in a volume of interest $\Omega$, denoted $\mathrm{TKE}_{\text {sum }}$ in the study of Ha et al, is calculated as TKE sum $=\int_{\Omega} \operatorname{TKE} d V[\mathrm{~J}]$, where TKE $\left[\mathrm{J} / \mathrm{m}^{3}\right]$ is computed based on the intravoxel velocity standard deviation (IVSD, $\sigma$ ) in three perpendicular directions [3],

$$
\mathrm{TKE}=\frac{\rho}{2} \sum_{i=1: 3} \sigma_{i}^{2} \quad \text { [Equation 1]. }
$$

When estimated on the basis of a non-symmetric motion-encoding scheme, the IVSD is computed as

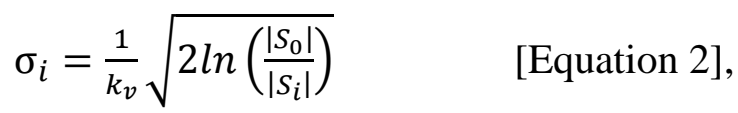

where $S_{i}$ is the MR-signal as a function of applied motion sensitivity, $k_{v}=\pi /$ VENC, in direction $i$ [4]. The optimal sensitivity is obtained when $S_{i} / S_{0}=0.6$, which corresponds to VENC $=\pi \tilde{\sigma}$, where $\tilde{\sigma}$ is the IVSD value of interest. If the VENC is excessively low relative to the spread of velocities with the voxel, $\left|S_{i}\right|$ approaches zero. However, due to the fact that MR magnitude data is Rician distributed, $\left|S_{i}\right|$ will be overestimated, and thus the IVSD will be underestimated. This effect is seen for the lowest VENC-values used in Figure $3 \mathrm{~b}$ in Ha et al, where the MR-measured $\mathrm{TKE}_{\text {sum }}$ is smaller than the CFD-derived $\mathrm{TKE}_{\text {sum. }}$ A high VENC, on the other hand, results a smaller difference in signal amplitude between $\left|S_{0}\right|$ and $\left|S_{i}\right|$ [2]. Consequently, when the VENC is excessively high relative to the spread of velocities with the voxel, the ratio $\left|S_{0}\right| /\left|S_{i}\right|$ should be close to one. However, noise will result in $\left|S_{0}\right| /\left|S_{i}\right|$ ratios that 
are sometimes smaller than one, and sometimes larger than one. When $\left|S_{0}\right| /\left|S_{i}\right|$ is smaller than one, the IVSD, if calculated from Equation 2, will become complexvalued. Ha et al addressed this situation by setting $\sigma$ to zero when $\left|S_{0}\right|<\left|S_{i}\right|$. By doing this, noise effects for $\left|S_{0}\right|<\left|S_{i}\right|$ are neglected, but noise effects for $\left|S_{0}\right|>\left|S_{i}\right|$ are included. This seems to be the source of the VENC-related bias seen in TKE $E_{\text {sum }}$ in the study of Ha et al. By calculating TKE directly from $\sigma^{2}$, where $\sigma^{2}$ is calculated as

$$
\sigma_{i}^{2}=\frac{2}{k_{v}{ }^{2}} \ln \left(\frac{\left|S_{0}\right|}{\left|S_{i}\right|}\right) \quad \text { [Equation 3], }
$$

this problem is avoided. In this way, positive and negative noise contributions will cancel out when computing $\mathrm{TKE}_{\text {sum }}$ with Equation 1. We believe that this would alter the appearance of Figures $3 b$ and $3 c$ in Ha et al and eliminate the VENC related positive bias in $\mathrm{TKE}_{\text {sum }}$ and $\mathrm{TKE}_{\text {noise }}$.

\section{References}

1. Ha, H., Hwang, D., Kim, G. B., Kweon, J., Lee, S. J., Baek, J., ... \& Yang, D. H. Estimation of Turbulent Kinetic Energy using 4D Phase-Contrast MRI: Effect of Scan Parameters and Target Vessel Size. Magnetic resonance imaging, 2016; In Press.

2. Dyverfeldt, P., Gårdhagen, R., Sigfridsson, A., Karlsson, M., \& Ebbers, T. On MRI turbulence quantification. Magnetic resonance imaging, 2009; 27(7), 913922.

3. Dyverfeldt, P., Kvitting, J. P. E., Sigfridsson, A., Engvall, J., Bolger, A. F., \& Ebbers, T. Assessment of fluctuating velocities in disturbed cardiovascular blood flow: In vivo feasibility of generalized phase- contrast MRI. Journal of Magnetic Resonance Imaging, 2008; 28(3), 655-663.

4. Dyverfeldt, P., Sigfridsson, A., Kvitting, J. P. E., \& Ebbers, T. Quantification of intravoxel velocity standard deviation and turbulence intensity by generalizing phase- contrast MRI. Magnetic resonance in medicine, 2006; 56(4), 850-858. 